\title{
Laboratorios de periodismo en España. Nuevas narrativas $y$ retos de futuro
}

institucional.us.es/ambitos/

\author{
Antonio López Hidalgo \\ Universidad de Sevilla \\ lopezhidalgo@us.es \\ María José Ufarte Ruiz \\ Universidad de Sevilla \\ mufarte@us.es
}

English Version: Laboratories journalism in Spain. New narratives and future challenges

\section{Resumen}

Este trabajo de investigación analiza el origen, la estructura, el funcionamiento y el desarrollo de los laboratorios de periodismo en España, que están creando nuevas fórmulas narrativas y otras maneras de informar y comunicar, mezclando distintos lenguajes y diferentes tecnologías. Desde el punto de vista metodológico, optamos por un método multidisciplinar que combina técnicas de investigación cualitativas y cuantitativas. En primer lugar, recurrimos a una imprescindible revisión bibliográfica para el establecimiento de un marco teórico pertinente, técnica que se completa con

otros procedimientos como las entrevistas estructuradas y los estudios de casos

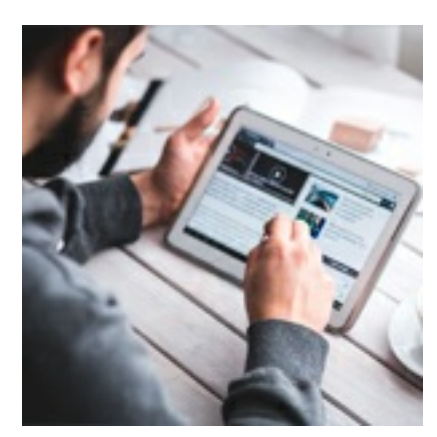
múltiples, donde los aspectos cualitativos se completan con un análisis cuantitativo

que nos permite extraer conclusiones y aportar ejemplos de interés a la investigación. La crisis que sufre la profesión periodística ha llevado a las empresas informativas a indagar en nuevos productos frente a otros ya obsoletos y cada vez menos rentables. Nacen así los cuatro laboratorios de periodismo que hay en España, que afrontan la innovación como principal reto de futuro.

\section{Palabras clave}

Laboratorio de periodismo, labs, innovación, internet, narrativas transmediáticas.

This research analyzes the origin, structure, operation and development laboratories journalism in Spain, which are creating new narrative formulas and other ways to inform and communicate, mixing different languages and different technologies. From the methodological point of view, we opted for a multidisciplinary approach that combines techniques of qualitative and quantitative research. First, we turn to an essential literature review for the establishment of a relevant theoretical framework, technical complete with other procedures such as structured interviews and studies of multiple cases where the qualitative aspects are complemented by a quantitative analysis that allows us draw conclusions and provide examples of interest to the investigation. The crisis in journalism has been informative to look into new products over other companies obsolete and less profitable. And four laboratories are born journalism in Spain, facing innovation as a major challenge for the

future.

Keywords

Laboratory journalism, labs, innovation, internet, transmedia narratives. 


\section{INTRODUCCIÓN}

El futuro del periodismo dibuja un paisaje deshecho y desdibujado. Pero hay que precisar: esta crisis no es nueva. No es fruto de la aparición de internet en nuestras vidas ni de la crisis de 2008. Como escribe Julia Cagé, en un libro hoy imprescindible, Salvar los medios de comunicación, tendemos a olvidarlo, pero ante cada innovación tecnológica -desde la radio, pasando por la televisión- la prensa escrita, y luego el conjunto de nuevos medios competidores, siempre han protestado, dando voces de alerta y augurado una muerte segura. Sin embargo, sí es cierto que esta crisis se ha acentuado en los próximos años. Los medios de comunicación tradicionales están ahora amenazados, desesperados. La información -cuya producción es muy costosa- se produce sin pagar.

Desde luego, hay otro factor que también influye y es que la gente comienza a sentirse insatisfecha con el contenido de los medios tradicionales. Algunas investigaciones demuestran que los reporteros españoles han evolucionado hacia un periodismo menos informativo, más trivial y sensacionalista, en el que la investigación queda relegada a un lugar secundario. Además, buena parte de la información que se publica en los medios procede de agencias de noticias, de oficinas de prensa de las administraciones o instituciones y distintos centros de documentación.

Pero es el trabajo de los periodistas lo que transforma un "hecho" en información. Definir la información es definir a quienes la producen: los periodistas. En tiempos de la cultura digital, de la información en tiempo real, de los blogs y las redes sociales, se lee a veces -equivocadamente- que hay tantos periodistas como internautas. Y se olvida que ser periodista es un oficio.

En este sentido, Julia Cagé ha dado un paso adelante para salvar a los medios de comunicación. Propone en particular la creación de un estatuto de "sociedad de medios de comunicación sin ánimo de lucro", a medio camino entre el estatuto de fundación y el de sociedad por acciones. Un modelo inspirado en parte en experiencias exitosas en las últimas décadas en el terreno de los medios de comunicación, así como en el de las grandes universidades internacionales, y que concilia actividad comercial y sin ánimo de lucro. Este ambicioso proyecto, señala esta autora, permite a la vez garantizar la financiación de los actores mediáticos congelando su capital y limitar el poder de decisión de los accionistas exteriores mediante la adopción de estatutos restrictivos. Ofrece un nuevo lugar a las sociedades de lectores y de trabajadores, y un marco jurídico y fiscal favorable al desarrollo de la financiación participativa. Los medios de comunicación -asegura Cagé- no pueden seguir siendo un terreno de juego para empresarios que quieren permitirse caprichos o "queridas", ni tampoco una inversión para especuladores en busca de chollos. Es la información como bien público -concluyela que constituye el pilar de la participación política y de la democracia (Cagé, 2015: 19-20).

Mientras tanto, algunas empresas tampoco se han quedado quietas a ver pasar el temporal y han comenzado a indagar nuevos productos frente a otros ya obsoletos y nada rentables. Un contexto cuyas características ya conocemos sobradamente: bajos salarios, precariedad laboral, continuos cierres de medios y creación de otros de vida efímera, los excesos del periodismo de mesa, el abuso de las fuentes institucionales, la sobreabundancia de informaciones, la gestación de nuevas rutinas productivas al hilo del desarrollo del periodismo en línea, la proliferación de contenidos basura, multiplicada por los agregadores de contenidos y, en definitiva, el agotamiento de los modelos tradicionales de periodismo.

Ante este contexto, algunas empresas comenzaron a crear laboratorios de periodismo buscando la piedra filosofal del futuro, nuevos lenguajes y otras maneras de informar, mezclando distintos lenguajes y diferentes tecnologías. Los resultados comienzan a ser asombrosamente sorprendentes. Quién lo podría pensar: el periodismo que contará el futuro aún está por inventar.

\section{METODOLOGÍA}

Desde el punto de vista metodológico, optamos por un método multidisplinar que combina técnicas de investigación cualitativas y cuantitativas. En primer lugar, recurrimos a una imprescindible revisión bibliográfica relativa a los laboratorios de periodismo con el objetivo de establecer un marco teórico de referencia. Para ello, 
acudimos a estudios académicos que han analizado la cuestión desde distintas perspectivas, como la corporativa y funcional, patente en la obra de Ramón Salaverría, Los Labs como fórmulas de innovación en los medios; o la profesional, reflejada en el artículo de Kevin Morán, Periodistas se encuentran en un laboratorio para crear vídeos inmersivos. La revisión bibliográfica se ha completado con un análisis de las reflexiones más destacadas encontradas en la Red, tanto en páginas webs como en blogs, de manera que podamos subsanar la ausencia de material bibliográfico para construir un corpus teórico referido a los laboratorios como nuevas fórmulas narrativas y retos de futuro.

Para alcanzar los objetivos fijados, completamos la revisión bibliográfica con otras dos técnicas propias de la investigación cualitativa: las entrevistas estructuradas y el estudio de casos múltiples, técnica en la que los aspectos cualitativos se completan con un análisis cuantitativo.

En lo referente a las entrevistas estructuradas, hemos elaborado un breve cuestionario estandarizado que plantea idénticas preguntas y en el mismo orden a los responsables de los cuatro laboratorios de periodismo, también conocidos como labs, que hay actualmente en España: Borja Bergareche, director de Innovación de Vocento; Alfredo Casares Corrales, fundador y director del Laboratorio de Innovación Periodística de Diario de Navarra (DN Lab); Miriam Hernanz, responsable del Laboratorio de RTVE; y Alejandro Lasso, director de Innovación y Estrategia de El Confidencial y responsable de EIConfidencial.LAB. De esta manera, conoceremos las distintas impresiones de estos profesionales acerca de algunas cuestiones que afectan a nuestro objeto de estudio, como el auge de esta nueva fórmula de innovación y la importancia para las empresas periodísticas. A éstos se les enviaron las siguientes preguntas:

- ¿Cuándo nace el Laboratorio?

- ¿Quién lo dirige?

- ¿Cuáles son las funciones u objetivos del Laboratorio?

- ¿Cuántas personas integran el LAB y cuáles son sus perfiles?

- ¿EI LAB es un departamento integrado dentro del organigrama empresarial o se encuentra al margen de éste?

- ¿Cómo está compuesto el organigrama?

- ¿Por qué es importante el Laboratorio para el medio o el grupo?

- ¿Con qué productos informativos trabaja?

- ¿Cómo ve el futuro del Laboratorio?

- ¿Cómo cree que serán los futuros productos informativos?

Por otro lado, hemos aplicado el estudio de casos múltiples paralelos, denominado así por Coller (200: 44), que implica la recolección y análisis de datos cualitativos y cuantitativos, con la finalidad de comprender, contrastar y profundizar en las cuestiones objeto de estudio. Esta técnica se basa en el razonamiento inductivo, pues las generalizaciones y teorías surgen de un proceso de indagación caracterizado por el examen sistemático y en profundidad de casos únicos. Así lo entienden, por ejemplo, Stake (1994: 236-247), que opina que, a través de este instrumento, el investigador puede alcanzar una mayor comprensión de uno particular, o bien el profesor Caro González, que escribe: "En el diseño de casos múltiples se utilizan varios casos únicos a la vez para estudiar la realidad que se desea explorar, describir, explicar, evaluar o modificar" (2004: 34).

En la investigación, hemos seleccionado cuatro casos posibles de la muestra, que corresponden a los cuatro labs que hay en España, es decir, el número total de unidades que conforman el universo a analizar. Los casos son tratados como una serie de experimentos y cada uno de ellos sirve para confirmar o negar las inferencias aportadas por los demás o las perspectivas conceptuales emergentes. Seguimos, por tanto, las recomendaciones de Eisenhardt (1984: 545), que habla de entre cuatro y diez casos, al considerar que con 
menos de cuatro es difícil generar teoría con mucha complejidad; y que con más de diez se excede la función de réplica que sustenta el método. En nuestro caso, los cuatro laboratorios existentes en España no son una muestra, sino que corresponden al universo total.

\section{LABORATORIOS DE PERIODISMO EN ESPAÑA}

En 2014, aunque su germen se sitúe a comienzos de la década, como bien señala Ramón Salaverría (2015a), cobró fuerza la implantación de laboratorios de innovación en el seno de los medios de comunicación. Sin hacer ruido, medios internacionales de referencia como The New York Times, The WaTshington Post, The Boston Globe, BBC, AFP, The Huffington Post o The Guardian comenzaron a abrir unidades y departamentos para experimentar en la creación de nuevos productos y servicios para hacer frente a los retos de futuro y poner pie en pared a una crisis que no desistía en su empeño de robar lectores y descapitalizar la industria periodística. La necesidad de encontrar productos innovadores y lenguajes con los que empatar con las audiencias se ha planteado desde entonces como un obstáculo a solventar buscando herramientas y tecnologías que abran paso a un futuro más esperanzador. En este ámbito, los laboratorios de periodismo deben afrontar la innovación como el principal reto de futuro.

Salaverría (2015b: 403) clasifica los tipos de laboratorios de periodismo en las empresas periodísticas en cuatro modelos. El primer modelo se centra en el desarrollo de tecnologías y aplicaciones digitales para los medios. Estos laboratorios, explica este autor, no son unidades de apoyo técnico para el trabajo cotidiano de los medios, sino departamentos que exploran nuevas oportunidades para la empresa. El segundo modelo engloba a aquellos laboratorios orientados a la innovación en lenguajes y a la creación de nuevas narrativas transmediáticas, al mismo tiempo que prestan una especial atención a los trabajos infográficos y al periodismo apoyado en base de datos. El tercer modelo se centra en promover proyectos empresariales e iniciativas comerciales, fórmula que las empresas añoran desde los años 1990 con vistas a aportar soluciones a ese continuo desafío. El cuarto modelo, según Salaverría, es el más alejado del concepto clásico de laboratorio, pues corresponde a centros dedicados a la formación de periodistas o al fomento de la alfabetización mediática, especialmente entre los jóvenes, y cuya denominación de laboratorios responde más a propósitos mercadotécnicos.

España cuenta con cuatro laboratorios de periodismo. El primero, RTVE.es Lab nace en 2012, creado por Radio Televisión Española, cuya página web es http://lab.rtve.es, y su twitter, @lab_rtvees. El segundo, DN Lab, lo crea Diario de Navarra en 2013. Su página web es http://laboratorio.diariodenavarra.es, y su twitter, @DNLaboratorio. El tercero, ElConfidencial.Lab, lo crea El Confidencial en 2014. No tiene página web y su twitter en @ECLaboratorio. Este mismo año el grupo Vocento crea Vocento Media Lab. Tampoco tiene página web y su twitter es @VocentoLab.

Además de estos cuatro laboratorios que es la materia de nuestro estudio, existe un quinto laboratorio o, lo que es lo mismo, un laboratorio de laboratorios, posiblemente de vida efímera, pero necesario de reseñar en esta investigación. Inmersive Journalisme Lab es el primer laboratorio de periodismo inmersivo creado en España. El laboratorio está impulsado por The App Date y cuenta con la participación de estos medios españoles: TVE, Cadena SER, Vocento y ABC, El País, La Sexta, Cuatro, El Mundo, Grupo Zeta y Diario Sport, Eldiario.es y Yorokobu. Los periodistas de los diferentes medios estarán encargados de guionizar y grabar piezas de forma conjunta con el impulso de la productora Virtual Natives. El blog del laboratorio de RTVE recoge así, fruto de este proceso, las claves más básicas para rodar un reportaje periodístico en 360 grados. VReak es la primera aplicación de noticias inmersivas en español y ya está disponible para Android e iOS. El ciudadano puede disfrutar de estas historias inmersivas directamente desde el teléfono móvil o bien con un dispositivo de realidad virtual con el que aumentar la sensación de inmersión.

Los periodistas españoles que han participado en este laboratorio han hecho públicas las diferencias entre un rodaje con realidad virtual y uno tradicional. Cuentan que el espacio importa tanto como la historia, pues el usuario necesita que el espacio le transmita sensaciones; el guión no debe estar saturado de información, ya que obstruye las sensaciones al vivir en una realidad de 360 grados; la historia debe ser colaborativa entre todo 
el equipo; hay que perder el miedo a cruzar lenguajes y aprovechar las transiciones entre planos para incorporar grafismos, pero para preservar el concepto inmersivo hay que incrustar también audios que arropen ese efecto; $y$, en último lugar, que la pieza tenga una duración de entre dos y tres minutos y con poco movimiento, para que el usuario no termine con una sensación de mareo (Hernanz, 2016).

Estos formatos muestran aún historias muy simples, pero ya se perciben las posibilidades narrativas que muestran. Un reto para los periodistas del futuro más próximo. Los profesionales tendrán que aprender a escribir y grabar para que sus historias puedan ser vividas por los ciudadanos. El periodismo inmersivo llevará al espectador al centro de la noticia, lo ubicará en mitad del conflicto elegido, le proporcionará una visión medida y exacta de los hechos y todavía aun podrá optar por conocer lugares y momentos que nunca hubiera soñado vivir, y todo gracias a su campo de visión de 360 grados. Miriam Hernanz, responsable de RTVE.es Lab, señala que en este sentido que "Inmersive Journalism Lab es una iniciativa de The App Date puntual, en la que distintos medios de comunicación nos unimos para desarrollar un reportaje inmersivo a través de un rodaje de VR. En concreto, el lab trabajó con Yorokobu, El País Vídeo y Vocento para desarrollar un reportaje en VR sobre la apicultura urbana".

\subsection{Vocento Media Lab: Narrativas interactivas y periodismo de datos}

Vocento Media Lab nace el 10 de diciembre de 2014. Con un curso de periodismo de datos a periodistas del grupo coincidiendo con la entrada en vigor de la Ley de Transparencia en España. Al frente del mismo figura el periodista Borja Bergareche, en calidad de director de Innovación de Vocento. Se trata de un laboratorio de formación, experimentación e innovación para todo el grupo.

Sus objetivos principales se pueden resumir en cuatro puntos. En primer lugar, como ya se ha dicho, dar formación a los equipos de Vocento, tanto a periodistas, equipos de desarrollo, comerciales, etcétera. En segundo lugar, impulsar dinámicas de experimentación tipo hackathones, con el fin de que los profesionales de distintos equipos de la compañía puedan trabajar conjuntamente. El término hackathon hace referencia al encuentro o evento entre programadores cuyo objetivo es el desarrollo colaborativo de software, aunque en ocasiones se puede encontrar también un componente de hardware. El término integra los conceptos de maratón y hacker, aludiendo a una experiencia colectiva que persigue la meta común de desarrollar y avanzar en el desarrollo de un proyecto, a base de involucrar a la comunidad en los mismos. Desde el punto de vista organizativo, supone una dinámica horizontal e intensiva en donde los participantes complementan experiencias y habilidades individuales con el propósito de desarrollar soluciones concretas.

El tercer objetivo se centra en mantener una relación estrecha con el talento externo y hacer de observatorio de startups. Al observatorio de startups también se le denomina observatorio de arranque, incipiente o simplemente emergente. Es un término utilizado actualmente en el mundo empresarial y hace referencia a ideas de negocios que están empezando o están en construcción. Generalmente se utiliza para empresas que innovan en el mercado y que están asociadas a la innovación, al desarrollo de tecnologías, al diseño o desarrollo web.

En cuarto lugar, se trata de un laboratorio de experimentación en el desarrollo de determinados productos. Un ejemplo es Eslang.es, un nuevo portal dirigido a millennials, también conocidos como generación Y o generación del milenio. El periodista A. Fernández S. (2012) los describe en ABC como los menores de 30 años, egocéntricos, muy listos y preparados académicamente que nacieron bajo el paraguas de la prosperidad económica. Antoni Gutiérrez-Rubí (2014) se pronuncia en la revista Forbes en la misma línea: "La generación millennials define a los nacidos entre 1981 y 1995, jóvenes entre 20 y 35 años que se hicieron adultos con el cambio de milenio (en plena prosperidad económica antes de la crisis). Son, por tanto, la futura generación de consumidores y usuarios, un mercado sustancial con nuevas características, necesidades y demandas que conviene conocer por las repercusiones y transformaciones que exigirá a las empresas". Por otra parte, el eslang o slang es un equipo que cumple con la legislación vigente en materia de protección de datos personales, pues la privacidad de los usuarios y la confidencialidad y seguridad de la información personal requiere la adopción de técnicas y medidas organizativas necesarias para evitar la pérdida, mal uso, alteración o el acceso no autorizado y robo de los datos personales facilitados. 
Asimismo, el área de Innovación de Vocento Media Lab cuenta con innovation engineer, es decir, ingeniería de la innovación, que se especializa en el diseño, desarrollo y mantenimiento de soluciones avanzadas de apoyo a la extracción y la gestión del conocimiento dentro de las organizaciones y en la web. El equipo de Estang lo constituyen tres periodistas y un desarrollador. Además, Vocentro Media Lab cuenta con dos becarios para investigar y desarrollar nuevas narrativas y periodismo de datos.

Este laboratorio está integrado dentro del organigrama del grupo empresarial y depende directamente de la Dirección de Innovación, que se encuadra en el área de Estrategia Digital de la compañía. En cuanto a la importancia del laboratorio para el futuro del grupo Vocente, Borja Bergareche entiende que éste es clave "para tener un ámbito muy orientado a la innovación en los objetivos y, sobre todo, en las formas de trabajo (agile metodologías ágiles y desarrollo de software ágil- y design thinking -pensamiento de diseño, analiza problemas complejos y los resuelve colectivamente-), que sirva de acelerador de tendencias clave para el negocio como las redes sociales, el video, las nuevas narrativas, la I+D y las nuevas formas de negocio y monetización, y que además actúe como un evangelizador interno sobre la transformación digital con una orientación muy a resultados y a 'quick gains' por eso de predicar con el ejemplo, y de ayudar realmente -y no solo mediante un cierto discurso sobre la innovación- a las redacciones y a los distintos equipos".

En cuanto a contenidos informativos, Vocento Media Lab ha trabajado hasta el momento, sobre todo, las nuevas narrativas interactivas y el periodismo de datos. En 2016 han comenzado a desarrollar más específicamente las narrativas audiovisuales. Valgan estos ejemplos:

http://www.abc.es/deportes/tenis/20150528/abci-nadal-campeon-tenis-201505281250.html

http://www.abc.es/realmadrid/cristiano-ronaldo-leyenda-20151001/; http://www.abc.es/deportes-chamberi

http://www.abc.es/madrid/20150519/abci-gastos-ayuntamiento-madrid-20150510090

http://especial-incendio.eldiariomontanes.es/recorrido-incendio-santander.html

http://www.abc.es/gasolineras

En definitiva, el laboratorio de Grupo Vocento está concebido como un proyecto experimental con respecto a su propia función y naturaleza. Media Lab significa, según su director, "cosas muy diferentes en distintos sitios". Y concluye: "Nosotros sabemos ahora que queremos darle las orientaciones apuntadas, con mucho foco hacia la capacitación interna. En el futuro lo iremos viendo".

\subsection{Laboratorio de Innovación Periodística de Diario de Navara (DN Lab): Apostando por productos digitales, móviles y personalizados}

El Laboratorio de Innovación Periodística de Diario de Navarra (DN Lab) nació en octubre de 2013. Lo funda y dirige el periodista Alfredo Casares Corrales, que es director de nuevos contenidos del Grupo La Información. DN Lab se divide en dos áreas, con sus respectivas funciones:

La primera de ellas, es la de periodismo de datos, visualización y nuevas narrativas audiovisuales. Se trata de un equipo multidisciplinar de periodista-programador-diseñador encargado de la elaboración de reportajes multimedia a través del uso de bases de datos y la incorporación de visualizaciones interactivas. Área donde se experimentan nuevos formatos periodísticos y narrativas audiovisuales, principalmente para el soporte digital. El área de periodismo de datos de DN Lab trabaja reportajes multimedia compuestos por visualizaciones interactivas en d3, líneas de tiempo, mapas interactivos, vídeos, infografías interactivas, galerías de imágenes, nubes de palabras, entre otros.

Y la segunda, proyectos de innovación y nuevos productos. Esta área de Investigación y Desarrollo cuenta entre sus funciones la elaboración de nuevos productos y servicios periodísticos, apoyados en metodologías ágiles como Design Thinking, Lean Startup y modelo Canvas. Esta área trabaja en equipos multidisciplinares compuestos por profesionales y alumnos de Sociología, Administración de Empresas, ingenieros en Informática y Telecomunicaciones, entre otros perfiles. 
Cinco personas integran DN Lab. Además, otros cinco alumnos forman parte actualmente de la experiencia del Laboratorio, cifra que varía conforme al número de alumnos que realiza cada semestre sus prácticas dentro de los equipos multidisciplinares ya descritos. Entre los perfiles hay periodistas (3), sociólogos (3), Ingenieros Informáticos (3) y en Telecomunicaciones (1). El Laboratorio de Innovación Periodística es un espacio autónomo que trabaja de forma autónoma y separada a las redacciones impresa y digital. Es un entorno de aprendizaje y experimentación que forma parte de la unidad de generación de nuevos contenidos que está desarrollando Diario de Navarra. Su organigrama es el siguiente: Director, Alfredo Casares Corrales; Coordinadora, Ramaris Albert Trinidad; Líder del área de Educación; Yulen Garmendia Iparraguirre; Contrato en Prácticas de Sociología, Leila Chivite Matthews; Beca PIE (Posgrado inmersión empresarial) Ingeniería Informática, Gontzal Aparicio Sanpedro; y Beca PIE (Posgrado inmersión empresarial) Periodismo, Ana I. Martín Cordobés.

DN Lab es una apuesta estratégica del Grupo La Información en la búsqueda de nuevos contenidos de valor, sobre todo en el ámbito digital, aunque no exclusivamente. Es, según señala Alfredo Casares Corrales, director de DN Lab: una atalaya desde la que mirar el sector y las nuevas tendencias del periodismo digital; un motor de aprendizaje constante. Conocer y experimentar con herramientas digitales innovadoras y transmitir ese conocimiento a nivel interno; un interlocutor entre la empresa, emprendedores y profesionales innovadores; un lugar mental desde el que dar forma al futuro del periodismo, en el que la empresa gane ganemos ritmo y velocidad y supere las limitaciones propias de una empresa de gran tamaño; un espacio autónomo que incorpora diversidad de perfiles, profesionales y edades enfocado al trabajo en equipos multidisciplinares; sirve de herramienta para medir y evaluar a nivel interno los contenidos periodísticos que publica la marca Diario de Navarra; y también es un espacio donde se promueve la formación y la utilización de nuevos recursos y herramientas para crear contenidos innovadores.

Fundar el Laboratorio de Innovación Periodística supuso, añade Casares, "anticiparnos a un proceso general de transformación de los medios con la tranquilidad de que tenemos un negocio que va bien, y con la ambición de que podemos contribuir a dar forma a nuestro futuro. Esto es una inversión. Es un acto de responsabilidad con un futuro incierto, para ir dotándonos de recursos, conocimiento y capacidades. Nos estamos preparando para afrontar lo que sea que venga con garantías e ilusión".

Este laboratorio ha cumplido una función muy necesaria desde su nacimiento: ser motor de la innovación. Ahora entra en una fase enfocada en transferir a la organización aprendizajes y herramientas, y generar nuevos contenidos. El siguiente paso debería ser contagiar a la organización, y en un futuro que toda la empresa funcionara como un laboratorio. Ese es el objetivo y el sueño de su director, Alfredo Casares, que sintetiza el futuro en esta frase: "Las tendencias que vemos apuntan a que los futuros productos informativos serán digitales, móviles, personalizados, conectados con el entorno social de cada usuario y dotados de elementos de interacción y gamificación que los hagan más atractivos".

\subsection{Laboratorio de Periodismo de RTVE.es: Webdocs y realidad virtual}

El Laboratorio de Periodismo de RTVE.es nace en junio de 2011, con el objetivo de explorar nuevas narrativas audiovisuales y potenciar nuevos lenguajes que el desarrollo de la tecnología hace hoy posibles. En un primer momento, el RTVE.es Lab apostaba por nuevos enfoques a historias de siempre, como el multicámara de Sanfermines. A medida que pasaban los meses y el proyecto se consolidaba, el departamento apostó por nuevos géneros audiovisuales, como los documentales interactivos. En este sentido, hay que señalar que las funciones y objetivos del Laboratorio de RTVE.es son: indagar y explorar nuevas narrativas audiovisuales; explotar nuevas posibilidades tecnológicas para desarrollar historias y esparcir la innovación por toda la redacción, impregnando de ese espíritu al resto de contenidos.

En un inicio, el lab fue puesto en marcha por dos personas (un diseñador y un periodista). A día de hoy, cinco años después, son nueve personas las que se han ido incorporando a esta iniciativa: tres periodistas, un realizador, un diseñador y cuatro desarrolladores informáticos, que trabajan las ideas desde cero de forma conjunta. Es por ello que se sientan en la misma área para explorar todos los aspectos del desarrollo de forma conjunta. El laboratorio forma parte de la Unidad Factoría Digital y Laboratorio, que dependen de la Subdirección de Contenidos y Transmedia de RTVE.es. Está, por tanto, totalmente integrado dentro del organigrama de la empresa. 
Para Miriam Hernanz, responsable del Laboratorio de RTVE, el laboratorio es importante para la cadena porque posibilita estar a la vanguardia de las narrativas digitales, desarrollando contenidos que están mucho más cerca de los nuevos públicos de lo que lo están los contenidos tradicionales de TVE. Además, el contenido de valor y los numerosos premios que el Lab ha recibido en este tiempo reflejan el trabajo de marca que el lab hace en favor de la empresa. El lab trabaja de forma colaborativa con las secciones de la web de informativos, con especial atención a temas internacionales. Además, desarrolla contenidos en colaboración con programas informativos de TVE como Documentos TV o En Portada, implementando la versión digital de algunos de sus trabajos.

De cara a un futuro inmediato, y a día de hoy, el laboratorio está metido de lleno en su primera gran producción en Realidad Virtual, con cuatro documentales grabados en $360^{\circ}$ para ser consumidos con gafas de VR. Su implicación en distintos trabajos de series de TVE le otorga un papel importante en la estrategia transmedia de RTVE.es. Asimismo, Miriam Hernanz destaca la apuesta del lab por los webdocs, ya que es una de las pocas instituciones comprometidas con esta narrativa, lo que "nos hace ser un agente importante en el panorama de la industria del documental interactivo en España". Respecto a cómo cree que serán los futuros productos informativos, Hernanz piensa que el consumo será "el que dicte la forma de producir, siendo obviamente el contenido digital el rey en el desarrollo de la estrategia informativa de cualquier medio de aquí a corto plazo. En este sentido, los diarios ya se han integrado en esa forma de producción pero las televisiones pronto comenzarán a trabajar así. Los productos informativos serán en breve contenidos que se adaptarán al dispositivo en el que lo consumas, más allá del diseño, adaptaremos los lenguajes para que la experiencia del usuario sea la que dicte la historia".

\subsection{EIConfidencial.LAB: Storytelling con interactividad avanzada y periodismo inmersivo}

Este laboratorio nace en noviembre de 2013 como el área de innovación, estrategia de producto y nuevas narrativas de El Confidencial. Las personas que integran en un principio este área habían pasado por la redacción y conocían a la perfección el medio. El objetivo era separarse de la vorágine del día a día de los contenidos y trabajar con más perspectiva en la segunda velocidad y en la estrategia de producto. La creación del EIConfidencial.LAB supone convertir al medio en una empresa de tecnología sin perder su esencia. De hecho, el principal cambio que introdujo fue involucrar al departamento técnico en la estrategia de la compañía. El objetivo principal fue acelerar el salto digital de El Confidencial. Hasta ese momento, el periódico había pasado por una etapa que fue la creación de un producto informativo diferente, de mucha calidad y rigor, y por otra en la que se habían añadido nuevas secciones para completar la oferta informativa y atraer a nuevos lectores. Gracias a haber seguido esos pasos y en ese orden, El Confidencial se convierte en un producto de éxito, con una audiencia sólida con un $70 \%$ de 'parroquianos'. Es en ese instante cuando el consejero delegado de la empresa impulsa la creación de EIConfidencial.LAB. Es así como arranca la tercera etapa del periódico, en la que actualmente se encuentra y donde apuesta decididamente por la innovación y la tecnología.

El laboratorio de El Confidencial cuenta con cuatro equipos de trabajo, cada uno de ellos orientado a diferentes productos: CMS, web, apps, growth y nuevas narrativas (gráficos, vídeo y periodismo de datos). En total son más de 30 personas enfocadas a estos objetivos a corto, medio y largo plazo. Entre ellos, cuenta con periodistas, programadores, diseñadores, analistas, jefes de producto, desarrolladores de móvil, expertos en UX, desarrolladores de negocio y nuevas audiencias. En este sentido, el laboratorio aglutina perfiles multidisciplinares con visiones en áreas periféricas que contribuyan al core del diario (la información). Cada grupo trabaja de forma independiente hasta el punto de que son capaces de sacar productos por sí mismos. Alejandro Lasso, director de Innovación y Estrategia de El Confidencial y responsable de EIConfidencial.LAB, señala al respecto: "Es como si cada uno de esos cuatro equipos tocase un instrumento, que al sonar al mismo tiempo reproducen una pieza acústica perfecta. Trabajamos con metodologías agile, enfocando siempre los productos al usuario final, para definir productos que evolucionan desde la experimentación hacia su concepción de éxito. Lo hacemos planteando MVP (productos mínimos viables) que testamos y evolucionamos para concebir productos exitosos".

Este lab se encuentra integrado dentro del organigrama empresarial. En el mismo, se pueden diferenciar dos departamentos bien diferentes. De una parte, la redacción del periódico, que está enfocada a la generación de material informativo de calidad, la materia prima con la que "fidelizamos a los lectores", añade Lasso. Y de otra, 
el personal del departamento dedicado al apartado de producto e innovación, que se centra en que esos contenidos de calidad estén presentados en un continente adecuado para atender a las necesidades del usuario. Alejandro Lasso no duda de la importancia de este laboratorio para el futuro de la compañía: "Estamos en un entorno de enorme cambio. La empresa periodística, en particular, se encuentra en el final de una etapa y el principio de la siguiente. Vamos a vivir un cambio de modelo de negocio donde intervienen varios actores importantes como los OTT y donde la distribución y la tecnología van a tomar un protagonismo absoluto. Para entender este cambio, se necesita una unidad como el laboratorio que sea capaz de explorar, de fallar rápido y barato, de aprender. Sólo las empresas que estén preparados para explorar, entender y adaptarse a esta nueva realidad serán las que sobrevivan y ese es nuestro reto actualmente".

Respecto a los productos informativos que explora actualmente EIConfidencial.LAB, el director de Innovación y Estrategia de El Confidencial afirma: "Estamos trabajando principalmente en móvil, trabajando en ofrecer una experiencia de navegación personalizada a nuestros lectores dependiendo del dispositivo en el que se encuentre y de sus intereses. Esa será la base para poder empezar a desarrollar power apps o servicios avanzados más allá de lo informativo. Desde la unidad de formato, trabajamos mucho el storytelling con elementos interactivos avanzados, vídeo $360^{\circ}$ y periodismo inmersivo". Sobre el futuro de este laboratorio, Alejandro Lasso responde: "Creo que a medida que las acciones que se impulsen desde el ámbito de la innovación tengan un retorno positivo para la empresa, hará que nos expandamos más. Nuestro futuro pasa por seguir apostando por equipos multidisciplinares que seguirán teniendo en el ADN el método de ensayo-error. Si no innovas, lo hará la competencia y si te relajas te quedas fuera. La autocomplacencia es nuestro mayor enemigo". En relación a cómo cree que serán los futuros productos informativos, el responsable de EIConfidencial.LAB es contundente: "Estarán orientados al móvil con un lenguaje completamente diferente al que estamos acostumbrados. De hecho, las apps puramente informativas de los medios de comunicación van a dejar de tener valor (si es que en algún instante lo han tenido) en un plazo menor del que nos esperamos. Con el aterrizaje de Facebook Instant Articles, AMP, Apple News, Snapchat Discover y otros agregadores, el lector va a tener a su disposición una oferta informativa enorme en un entorno muy optimizado con el que los generadores de contenidos no podremos competir de forma individualizada. Nuestra oportunidad radica en trabajar en productos y servicios innovadores que el usuario no pueda encontrar en otro sitio, hacer la vida más cómoda al lector e ir ampliando nuestra mirada a otros sectores".

\section{DISCUSIÓN Y CONCLUSIONES}

En tiempos de crisis, sobre todo en tiempos de crisis para el periodismo, las empresas informativas no se han quedado quietas a ver pasar el temporal y han comenzado a indagar nuevos productos frente a otros ya obsoletos y nada rentables. Han abierto nuevas unidades y departamentos para experimentar en la creación de productos y servicios con el fin de hacer frente a los retos de futuro y poner pie en pared a una crisis que no desiste en su empeño de robar lectores y descapitalizar la industria periodística. Es así como surgen, especialmente a partir de 2014, los laboratorios de periodismo en el seno de los medios de comunicación españoles, que toman como modelo diferentes medios internacionales que ya se habían enfrascado en experiencias similares.

Dentro de los modelos de laboratorios que propone Salaverría, los cuatro existentes en España cabría encuadrarlos dentro de la segunda categoría; es decir, entre aquellos que se dedican a la innovación de nuevos productos informativos. DN LAB, EIConfidencial.Lab y Vocento Media Lab prestan especial importancia al periodismo de datos, posiblemente porque los tres trabajan el periodismo escrito. Los cuatro, en cambio, profundizan en la creación de nuevas narraciones interactivas. Asimismo, RTVE.es y EIConfidencial.Lab dan especial interés al video y audio grabado en $360^{\circ}$ y al periodismo inmersivo. Estos dos últimos laboratorios, junto con Vocento Media Lab, y otros medios españoles, se han integrado en el seno de Inmersive Journalisme Lab, impulsado por The App Date, primer laboratorio español dedicado a la investigación del periodismo inmersivo.

Los cuatro laboratorios están compuestos por perfiles multidisciplinares con visiones en áreas periféricas, que actúan como los diferentes elementos de una orquesta, donde "cada miembro toca un instrumento diferente que 
al sonar al mismo tiempo reproducen una pieza acústica perfecta", según Alejandro Lasso, responsable de EIConfidencial.LAB. Y sus consumidores potenciales, en opinión de Borja Bergareche, director de Innovación de Vocento, es un mercado sustancial con nuevas características, necesidades y demandas que conviene conocer por las repercusiones y transformaciones que exigirá a las empresas.

\section{REFERENCIAS}

CAGÉ, Julia (2015): Salvar los medios de comunicación. Anagrama, Barcelona.

CARO GONZÁLEZ, Francisco Javier (2002): Cambio e innovación en la empresa informativa. Grupo de Investigación, Gestión de la Innovación, la Calidad y el Cambio, Sevilla.

COLLER, Xavier (2000): Estudio de casos. Cuadernos Metodológicos, 30. Centro de Investigaciones Sociológicas, Madrid.

EISENHARDT, Kathleen M. (1989): Building Theories from Case Study Research.

The Academy of Management Review [en línea]. Vol. 14, n. 4, pp. 532-550. Fecha de consulta: 17/03/2016. Disponible en: https://www.jstor.org/stable/258557?seq=1\#page_scan_tab_contents

FERNÁNDEZ S., A. (2012): "Millenials: la generación malcriada que quiere cambiar el futuro" en $A B C, 6$ de noviembre de 2012. [en línea]. Fecha de consulta: 03/03/2016. Disponible en:

http://www.abc.es/20121103/sociedad/abci-millennials-generacion-201211021603.html.

GURIÉRREZ-RUBÍ, Antoni (2014): “6 rasgos clave de los millennials, los nuevos consumidores” en Forbes, 22 de diciembre de 2014 [en línea]. Fecha de consulta: 03/03/2016. Disponible en: http://www.forbes.com.mx/6rasgos-clave-de-los-millennials-los-nuevos-consumidores/.

MORÁN, Kevin (2016): "Periodistas se encuentran en un laboratorio para crear vídeos inmersivos", 10 de marzo de 2016. [en línea]. Fecha de consulta: 03/03/2016. Disponible en:

http://www.clasesdeperiodismo.com/2016/01/18/espana-periodistas-se-encuentran-en-un-laboratorio-paracrear-videos-inmersivos/.

SALAVERRÍA, Ramón (2015a): "Periodismo en 2014: balance y tendencias".

http://www.cuadernosdeperiodistas.com/media/2015/01/09-22-SALAVERRIA.pdf.

SALAVERRÍA, Ramón (2015b): “Los labs como fórmula de innovación en los medios”. El profesional de la información, julio-agosto. V. 24. N. 4. ISSN: 1699-2407.

STAKE, Robert E (1994): "Case Studies" en Norman K. DENZIN y LINCOLN Yvonna S (eds.). Handbook of qualitative research. Thousands Oak Sage Publications, Estados Unidos.

STAKE, Robert E. (1995): Investigación con estudio de casos. Morata, Madrid.

STONE, M. (2001): "Los pasos que conducen al periodismo multimedia" en GINER Juan Antonio GINER y SUSSMAN Barry (eds.): Innovaciones en periódicos: informe mundial 2000: [un estudio anual del Innovation International Media Consulting Group para la Asociación Mundial de Periódicos]. Pamplona Innovation, Pamplona.

TERCEIRO LOMBA, José B. (1996): Sociedad digital: del homo sapiens al homo digitalis. Alianza, Madrid.

TERCEIRO LOMBA, José B.; CLAVERO, Gustavo Matías (2001): Digitalismo: el nuevo horizonte sociocultural. Taurus Ediciones, Madrid.

UFARTE RUIZ, María José (2016): Entrevista realizada a Borja Bergareche. (11.01.2016). 
UFARTE RUIZ, María José (2016): Entrevista realizada a Alfredo Casares. (19.02.2016).

UFARTE RUIZ, María José (2016): Entrevista realizada a Miriam Hernanz. (07.04.2016).

UFARTE RUIZ, María José (2016): Entrevista realizada a Alejandro Lasso. (27.04.2016).

WILDEMUTH, Barbara M. (2009): Applications of social research methods to questions in information and library science. Westport Libraries Unlimited, Estados Unidos.

WOLF, Mauro (1991): La investigación de la comunicación de masas: Crítica y perpectivas . 2a ed. Paidós, Barcelona.

\section{BREVE SEMBLANZA DE LOS AUTORES}

María José Ufarte (Almería, 1983) es licenciada en Periodismo por la Universidad de Sevilla. Doctora en Periodismo desde el año 2001 por esta misma Universidad con la Tesis titulada El periodista acosado: Entre la precariedad laboral y el mobbing. Un estudio de caso: La precariedad de los periodistas almerienses en la prensa escrita, publicada por la Universidad Hispalense. Como periodista, ha trabajado en prensa escrita, gabinetes de comunicación y agencias especializadas en consultoría estratégica. Sus artículos de investigación han sido publicados en prestigiosas revistas especializadas, como Estudios sobre el mensaje periodístico y Ámbitos Comunicación, entre otras.

Antonio López Hidalgo (Montilla, Córdoba, 1957) es periodista y profesor titular de la Facultad de Comunicación de la Universidad de Sevilla. Licenciado en Ciencias de la Información por la Universidad Complutense de Madrid y Doctor en Periodismo por la Universidad Hispalense. En 1990 publica su primera obra periodística junto a Juan Emilio Ballesteros, El sindicato clandestino de la Guardia Civil, libro por el que fue procesado y posteriormente absuelto. Entre sus obras académicas destacan Géneros periodísticos complementarios. Una aproximación crítica a los formatos del periodismo visual, La Columna. Periodismo y Literatura en un género plural, Periodismo de inmersión para desenmascarar la realidad, escrito junto a María de los Ángeles Fernández Barrero.

Ámbitos. Revista Internacional de Comunicación, n.34, edición de otoño, 2016.

Recibido: 13/07/2016

Aprobado: 02/09/2016 